NASA/CR-1998-208712

ICASE Report No. 98-42

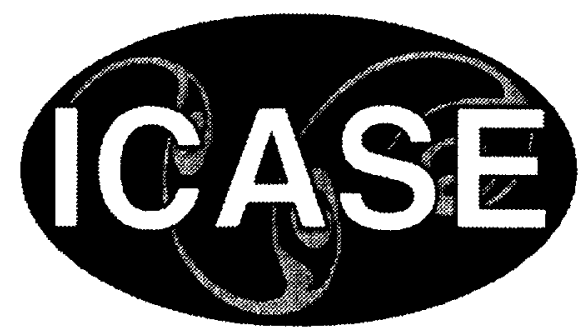

\title{
Real Gas Computation Using an Energy Relaxation Method and High-order WENO Schemes
}

Philippe Montarnal and Chi-Wang Shu

Brown University, Providence, Rhode Island

Institute for Computer Applications in Science and Engineering NASA Langley Research Center

Hampton, VA

Operated by Universities Space Research Association

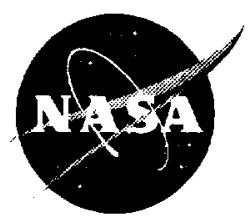

National Aeronautics and

Space Administration

Langley Research Center

Prepared for Langley Research Center

Hampton, Virginia 23681-2199 under Contract NAS1-97046 
Available from the following:

NASA Center for AeroSpace Information (CASI)

7121 Standard Drive

National Technical Information Service (NTIS)

Hanover, MD 21076-1320

(301) 621-0390

5285 Port Royal Roa 1

Springfield, VA 221 1 1-2171

(703) $487-4650$ 


\title{
REAL GAS COMPUTATION USING AN ENERGY RELAXATION METHOD AND HIGH-ORDER WENO SCHEMES
}

\author{
PHILIPPE MONTARNAL * AND CHI-WANG SHU ${ }^{\dagger}$
}

\begin{abstract}
In this paper, we use a recently developed energy relaxation theory by Coquel and Perthame and high order weighted essentially non-oscillatory (WENO) schemes to simulate the Euler equations of real gas. The main idea is an energy decomposition into two parts: one part is associated with a simpler pressure law and the other part (the nonlinear deviation) is convected with the flow. A relaxation process is performed for each time step to ensure that the original pressure law is satisfied. The necessary characteristic decomposition for the high order WENO schemes is performed on the characteristic fields based on the first part. The algorithm only calls for the original pressure law once per grid point per time step, without the need to compute its derivatives or any Riemann solvers. Both one and two dimensional numerical examples are shown to illustrate the effectiveness of this approach.
\end{abstract}

Key words. Euler equations, real gas, weighted essentially non-oscillatory schemes.

Subject classification. Applied and Numerical Mathematics

1. Introduction. In this paper we consider the Euler equations for a real compressible inviscid fluid,

$$
\mid \begin{aligned}
& \partial_{t} \rho+\operatorname{div}(\rho u)=0, \quad t \geq 0, x \in \mathbb{R}^{d} \\
& \partial_{t} \rho u+\operatorname{div}(\rho u \otimes u+p)=0 \\
& \partial_{t} E+\operatorname{div}((E+p) u)=0 \\
& E=\frac{1}{2} \rho|u|^{2}+\rho \varepsilon
\end{aligned}
$$

where the quantities $\rho, u, p, E$ and $\varepsilon$ represent the density, velocity, pressure, total encrgy and specific internal energy, respectively. In addition, there is an equation of state (EOS) of the form $p=p(\rho, \varepsilon)$ associated with a strictly convex entropy $\rho s(\rho, \varepsilon)$ which satisfies the following entropy inequalities

$$
\partial_{t} \rho s+\operatorname{div}(\rho s u) \leq 0
$$

The pressure law is furthermore assumed to satisfy

$$
p_{, \varepsilon}(\rho, \varepsilon)>0, p(\rho, 0)=0 \text { and } p(\rho, \infty)=\infty
$$

In the literature research has been done in order to extend classical schemes designed for perfect gas to real gases. Collela and Glaz [1] extended the numerical procedure for obtaining the exact Riemann solution to a real-gas case, Grossman and Walters [7], Liou, van Leer and Shuen [13] extended the method of fluxvector splitting and flux-difference splitting, Montagné, Yee and Vinokur [16] developed second-order explicit

*Division of Applied Mathematics, Brown University, Providence, RI 02912. Current address: Commissariat à l'Energie Atomique, Centre d'étude de Bruyères-le-Châtel, BP 12 - 91680 Bruyères le Châtel, France. E-mail: philippe.montarnal@bruyeres.cea.fr. Research supported by an INRIA post-doctoral grant while this author was in residence at Brown University.

${ }^{\dagger}$ Division of Applied Mathematics, Brown University, Providence, RI 02912. E-mail: shu@cfm.brown.edu. Research of this author was supported in part by ARO grant DAAG55-97-1-0318, NSF grant DMS-9500814, NASA Langley grant NAG-11145 and Contract NAS1-97046 while in residence at ICASE, NASA Langley Research Center, Hampton, VA 23681-2199, and AFOSR grant F49620-96-1-0150. 
shock-capturing schemes for real gas, Glaister [5] presented an extension of approximate linearized Riemann solver with different averaged matrices, while Loh and Liou $[15]$ used the generalization of their Lagrangian approach (originally proposed for perfect gas) to obtain the real gas Riemann solution.

Most of the previous proposed methods would require a computation of the pressure law and its derivatives, or a Riemann solver. This is not only costly but also problematic when there is no analytical expressions of the pressure law (for example if we have only table values).

Recently Coquel and Perthame [2] have introduced an energy relaxation theory for Euler equations of real gas. The main idea is to introduce a relaxation of the noninear pressure law by considering an energy decomposition under the form $\varepsilon=\varepsilon_{1}+\varepsilon_{2}$. The internal energy $\varepsilon_{1}$ is associated with a simpler pressure law $p_{1}$ (which is taken as the $\gamma$-law in this paper), while $\varepsilon_{2}$ stands for the nonlinear perturbation and is simply convected by the flow. These two energies are also subject to a relaxation process and in the limit of an infinite relaxation rate, one recovers the initial pressure law $p$.

From this general framework, Coquel and Perthame have also deduced the extension to general pressure laws of classical schemes for polytropic gases, which only uses a single call to the pressure law per grid point and time step. No derivatives of the pressure law or any Riemann solvers need to be computed. Another advantage of their approach is that its implementation does not depend on the particular expression of the equation of states. For the first order Godunov scheme, they have shown that this extension satisfies stability, entropy and accuracy conditions. Numerical examples have been provided using first order schemes by A. In $[9]$.

For Euler equations of polytropic gas, high order ENO (essentially non-oscillatory) and WENO (weighted ENO) schemes $[8,18,19,20,14,10,21]$ have been quite successful in providing high resolution results for complicated flow structures and shocks. The idea of ENO schemes is to adaptively choose local stencils so that interpolation across a discontinuity is avoided as much as possible. WENO uses a nonlinear combination of all stencils to improve upon accuracy and smoothness of numerical fluxes while maintaining the non-oscillatory behavior of ENO near a discontinuity.

The aim of this paper is to study the implementation of this relaxation method with high order WENO schemes [10] for real gases. One and two dimensional numerica examples will be given.

In Section 2 we provide the general framework of the energy relaxation theory of $[2]$, followed by a short description of high order WENO schemes [10]. We then give the details of the construction of the relaxed WENO schemes for general gases. In Section 3 numerical examples are given. We start with a description of the different equations of states used in this paper, followed by one dimensional shock tube test problems. Two dimensional test cases of a smooth vortex, to test the accuracy of the schemes, and of the double Mach reflection problem, are then presented. Concluding remarks ase given in Section 4 . In the appendices, we give the expressions of the Roe matrices for the relaxation syst am (A) and the two molecular vibrating gas (B).

\section{Implementation of the energy relaxation method with WENO.}

2.1. Energy relaxation theory. The principle of the er ergy relaxation theory developed by Coquel and Perthame [2] is to find a pressure law $p_{1}\left(\rho^{\lambda}, \varepsilon_{1}^{\lambda}\right)$ (simpler than $p$, typically a polytropic law) and an internal energy $\phi\left(\rho^{\lambda}, \varepsilon_{1}^{\lambda}\right)$ so that the system (1.1) and the entr spy inequality (1.2) can be recovered, in the limit of an infinite relaxation rate $\lambda$ (called the equilibrium limit), from the following system (called the 
relaxation system):

$$
\mid \begin{aligned}
& \partial_{t} \rho^{\lambda}+\operatorname{div}\left(\rho^{\lambda} u^{\lambda}\right)=0, \quad t \geq 0, x \in \mathbb{R}^{d} \\
& \partial_{t} \rho^{\lambda} u^{\lambda}+\operatorname{div}\left(\rho^{\lambda} u^{\lambda} \otimes u^{\lambda}+p_{1}^{\lambda}\right)=0 \\
& \partial_{t} E_{1}^{\lambda}+\operatorname{div}\left(\left(E_{1}^{\lambda}+p_{1}^{\lambda}\right) u^{\lambda}\right)=\lambda \rho^{\lambda}\left(\varepsilon_{2}^{\lambda}-\phi\left(\rho^{\lambda}, \varepsilon_{1}^{\lambda}\right)\right) \\
& \partial_{t} \rho^{\lambda} \varepsilon_{2}^{\lambda}+\operatorname{div}\left(\rho^{\lambda} u^{\lambda} \varepsilon_{2}^{\lambda}\right)=-\lambda \rho^{\lambda}\left(\varepsilon_{2}^{\lambda}-\phi\left(\rho^{\lambda}, \varepsilon_{1}^{\lambda}\right)\right), \\
& E_{1}^{\lambda}=\frac{1}{2} \rho^{\lambda}\left|u^{\lambda}\right|^{2}+\rho^{\lambda} \varepsilon_{1}^{\lambda},
\end{aligned}
$$

where $p_{1}\left(\rho^{\lambda}, \varepsilon_{1}^{\lambda}\right)=\left(\gamma_{1}-1\right) \rho^{\lambda} \varepsilon_{1}^{\lambda}$ with $\gamma_{1}$ a given constant greater than 1 . One can prove [2] that the relaxation system (2.1) can be supplemented by entropy inequalities under the form

$$
\partial_{t} \rho^{\lambda} \Sigma+\operatorname{div}\left(\rho^{\lambda} \Sigma u^{\lambda}\right) \leq \operatorname{RED}^{\lambda}:=-\lambda \rho^{\lambda}\left(\Sigma_{, s_{1}} s_{1, \varepsilon_{1}}-\Sigma_{, \varepsilon_{2}}\right)\left(\varepsilon_{2}-\phi\left(\rho^{\lambda}, \varepsilon_{1}^{\lambda}\right)\right)
$$

where $s_{1}\left(\rho, \varepsilon_{1}\right)=\rho^{\gamma_{1}-1} / \varepsilon_{1}$ and the specific entropy $\Sigma$ denotes an arbitrary function in $C^{1}\left(\mathbb{R}_{+}^{2}\right)$ such that $\rho \Sigma$ is convex in $\left(\rho, \rho \varepsilon_{1}, \rho \varepsilon_{2}\right)$ and that can be written under the form $\Sigma=\Sigma\left(s_{1}\left(\rho, \varepsilon_{1}\right), \varepsilon_{2}\right)$. RED $D^{\lambda}$ represents the Rate of Entropy Dissipation.

Formally, the original Euler system (1.1) will be recovered at $\lambda \rightarrow+\infty$ with

$$
\varepsilon=\varepsilon_{1}+\varepsilon_{2}=\varepsilon_{1}+\phi\left(\rho, \varepsilon_{1}\right)
$$

provided that we have the following condition (called the consistency condition)

$$
p\left(\rho, \varepsilon_{1}+\phi\left(\rho, \varepsilon_{1}\right)\right)=p_{1}\left(\rho, \varepsilon_{1}\right)=\left(\gamma_{1}-1\right) \rho \varepsilon_{1} .
$$

This last condition can be fulfilled for any given choice of $\gamma_{1}>1$.

But in addition to the conservative system (1.1), one also wants to recover at the limit the entropy inequality (1.2). The following result, due to Coquel and Perthame [2], gives this last condition under a characterization of the admissible $\gamma_{1}$.

Theorem 2.1. Assuming that $\gamma_{1}$ satisfies

$$
\begin{aligned}
& \gamma_{1}>\sup _{\rho, \varepsilon} \Gamma(\rho, \varepsilon) \text { where } \Gamma(\rho, \varepsilon)=1+\frac{p_{, \varepsilon}}{\rho} \text {, } \\
& \gamma_{1}>\sup _{\rho, \varepsilon} \gamma(\rho, \varepsilon) \text { where } \gamma(\rho, \varepsilon)=\frac{\rho}{p} p_{, \varepsilon}+\frac{p_{, \varepsilon}}{\rho} \text {, }
\end{aligned}
$$

provided that $\gamma_{1}$ is finite, we then have

(i) there exists a (unique) specific entropy $\Sigma\left(s_{1}, \varepsilon_{2}\right)$ such that at equilibrium $\left(\varepsilon=\varepsilon_{1}+\phi\left(\rho, \varepsilon_{1}\right)\right)$

$$
s(\rho, \varepsilon)=\Sigma\left(s_{1}\left(\rho, \varepsilon_{1}\right), \phi\left(\rho, \varepsilon_{1}\right)\right)
$$

(ii) this entropy is uniformly compatible with the relaxation procedure, i.e.:

$$
R E D^{\lambda} \leq 0, \text { for all } \lambda>0 \text {. }
$$

2.2. WENO schemes. We use the fifth order WENO scheme in [10]. For a scalar conservation law

$$
u_{t}+f(u)_{x}=0
$$

the derivative $f(u)_{x}$ at the grid point $x=x_{j}$ is approximated by a conservative flux difference

$$
\left.f(u)_{x}\right|_{x=x_{j}} \approx \frac{1}{\Delta x}\left(\hat{f}_{j+\frac{1}{2}}-\hat{f}_{j-\frac{1}{2}}\right) .
$$


The WENO numerical flux $\hat{f}_{j+\frac{1}{2}}$ is computed as follows. For a positive wind direction $f^{\prime}(u) \geq 0$, we first define 3 third order numerical fluxes:

$$
\begin{aligned}
& \hat{f}_{j+\frac{1}{2}}^{1}=\frac{1}{3} f\left(u_{j-2}\right)-\frac{7}{6} f\left(u_{j-1}\right)+\frac{11}{6} f\left(u_{j}\right), \\
& \hat{f}_{j+\frac{1}{2}}^{2}=-\frac{1}{6} f\left(u_{j-1}\right)+\frac{5}{6} f\left(u_{j}\right)+\frac{1}{3} f\left(u_{j+1}\right), \\
& \hat{f}_{j+\frac{1}{2}}^{3}=\frac{1}{3} f\left(u_{j}\right)+\frac{5}{6} f\left(u_{j+1}\right)-\frac{1}{6} f\left(u_{j+2}\right) .
\end{aligned}
$$

A third order ENO scheme will result if we choose one of the three third order fluxes in (2.7) adequately, according to the size of divided differences [19]. On the other hand, a fifth order linear scheme will result if we choose the flux as

$$
\hat{f}_{j+\frac{1}{2}}^{\text {linear }}=d_{1} \hat{f}_{j+\frac{1}{2}}^{1}+d_{2} \hat{f}_{j+\frac{1}{2}}^{2}+d_{3} \hat{f}_{j+\frac{1}{2}}^{3}
$$

with

$$
d_{1}=\frac{1}{10}, \quad d_{2}=\frac{3}{5}, \quad d_{3}=\frac{3}{10} .
$$

The fifth order WENO scheme results if we choose the numerical flux as

$$
\hat{f}_{j+\frac{1}{2}}=\omega_{1} \hat{f}_{j+\frac{1}{2}}^{1}+\omega_{2} \hat{f}_{j+\frac{1}{2}}^{2}+\omega_{3} \hat{f}_{j+\frac{1}{2}}^{3}
$$

with $\omega_{i}$ defined by

$$
\omega_{i}=\frac{\alpha_{i}}{\sum_{s=1}^{3} \alpha_{s}}, \quad \alpha_{i}=\frac{d_{i}}{\left(\epsilon+\beta_{i}\right)^{2}}
$$

and

$$
\begin{aligned}
& \beta_{1}=\frac{13}{12}\left(f\left(u_{i-2}\right)-2 f\left(u_{i-1}\right)+f\left(u_{i}\right)\right)^{2}+\frac{1}{4}\left(f\left(u_{i-2}\right)-4 f\left(u_{i-1}\right)+3 f\left(u_{i}\right)\right)^{2}, \\
& \beta_{2}=\frac{13}{12}\left(f\left(u_{i-1}\right)-2 f\left(u_{i}\right)+f\left(u_{i+1}\right)\right)^{2}+\frac{1}{4}\left(f\left(u_{i-1}\right)-f\left(u_{i+1}\right)\right)^{2}, \\
& \beta_{3}=\frac{13}{12}\left(f\left(u_{i}\right)-2 f\left(u_{i+1}\right)+f\left(u_{i+2}\right)\right)^{2}+\frac{1}{4}\left(3 f\left(u_{i}\right)-4 f\left(u_{i+1}\right)+f\left(u_{i+2}\right)\right)^{2} .
\end{aligned}
$$

$\epsilon$ in (2.11) is taken as $10^{-6}$ in all our numerical examples, as was done in [10]. The weights in (2.11) are chosen so that in smooth regions (including at smooth extrema), the WENO flux (2.10) behaves similarly as the linear flux (2.8) and is uniformly fifth order accurate. Niear shocks, however, the WENO flux (2.10) behaves similarly as an ENO flux, in the sense that any stercil crossing a discontinuity has a near zero weight. For details of the derivation, see $[10,21]$.

If the wind direction is negative, $f^{\prime}(u) \leq 0$, the procedure is symmetric to the case with $f^{\prime}(u) \geq 0$, with respect to the location $x_{j+\frac{1}{2}}$. In general, a flux splitting is used

$$
f(u)=f^{+}(u)+f^{-}(\iota)
$$

such that $f^{+}(u)$ has a positive wind direction and $f^{-}(u)$ has a negative wind direction:

$$
\frac{d}{d u} f^{+}(u) \geq 0, \quad \frac{d}{d u} f^{-}(u) \leq 0
$$

The procedure described above can then be applied to $f^{+}(\imath)$ and $f^{-}(u)$ separately. The simplest flux splitting is the Lax-Friedrichs splitting:

$$
f^{ \pm}(u)=\frac{1}{2}(f(u) \pm a u)
$$


where

$$
\alpha=\max _{u}\left|f^{\prime}(u)\right|
$$

Notice that while first and second order schemes with a Lax-Friedrichs splitting is quite dissipative, higher order schemes based on the Lax-Friedrichs fluxes usually give very good results. We use Lax-Friedrichs fluxes in this paper.

For systems of conservation laws in this paper, we use both a component-wise version, where the procedure described above is applied to each equation in the system separately, and a characteristic version, where locally we apply the procedure described above on characteristic projections. For details about how to perform a local characteristic procedure, see for example $[18,19,10]$.

2.3. Construction of the relaxed WENO scheme. The procedure to solve the Euler system (1.1) within the framework of the energy relaxation theory is the following. Given the numerical equilibrium solution at the time level $t^{n}$

$$
\rho\left(x, t^{n}\right), u\left(x, t^{n}\right), \varepsilon\left(x, t^{n}\right)
$$

this approximation is advanced to the next time level $t^{n+1}=t^{n}+\Delta t$ in two steps.

- First step: relaxation. The two internal energies $\varepsilon_{1}\left(x, t^{n}\right)$ and $\varepsilon_{2}\left(x, t^{n}\right)$ are obtained by $(2.2)$ and the consistency condition (2.3):

$$
\begin{aligned}
& \varepsilon_{1}\left(x, t^{n}\right)=\frac{p\left(\rho\left(x, t^{n}\right), \varepsilon\left(x, t^{n}\right)\right)}{\left(\gamma_{1}-1\right) \rho\left(x, t^{n}\right)} \\
& \varepsilon_{2}\left(x, t^{n}\right)=\varepsilon\left(x, t^{n}\right)-\varepsilon_{1}\left(x, t^{n}\right) .
\end{aligned}
$$

Notice that this step involves just one call to the pressure law per grid point and does not involve any derivatives of the pressure law or any iterations.

- Second step: evolution in time. For $t^{n} \leq t \leq t^{n+1}$, we solve the Cauchy problem for the relaxation system (2.1), with zero on the right side:

$$
\mid \begin{aligned}
& \partial_{t} \rho^{\lambda}+\operatorname{div}\left(\rho^{\lambda} u^{\lambda}\right)=0, \quad t \geq 0, x \in \mathbb{R}^{d} \\
& \partial_{t} \rho^{\lambda} u^{\lambda}+\operatorname{div}\left(\rho^{\lambda} u^{\lambda} \otimes u^{\lambda}+p_{1}^{\lambda}\right)=0 \\
& \partial_{t} E_{1}^{\lambda}+\operatorname{div}\left(\left(E_{1}^{\lambda}+p_{1}^{\lambda}\right) u^{\lambda}\right)=0 \\
& \partial_{t} \rho^{\lambda} \varepsilon_{2}^{\lambda}+\operatorname{div}\left(\rho^{\lambda} u^{\lambda} \varepsilon_{2}^{\lambda}\right)=0 \\
& E_{1}^{\lambda}=\frac{1}{2} \rho^{\lambda}\left|u^{\lambda}\right|^{2}+\rho^{\lambda} \varepsilon_{1}^{\lambda}
\end{aligned}
$$

and the initial data

$$
\rho\left(x, t^{n}\right), u\left(x, t^{n}\right), \varepsilon_{1}\left(x, t^{n}\right), \varepsilon_{2}\left(x, t^{n}\right)
$$

and we obtain at time $t^{n+1-}$

$$
\rho\left(x, t^{n+1-}\right), u\left(x, t^{n+1-}\right), \varepsilon_{1}\left(x, t^{n+1-}\right), \varepsilon_{2}\left(x, t^{n+1-}\right) .
$$

At last, we compute the equilibrium solution at time $t^{n+1}$ by

$$
\begin{aligned}
& \rho\left(x, t^{n+1}\right)=\rho\left(x, t^{n+1-}\right), \\
& u\left(x, t^{n+1}\right)=u\left(x, t^{n+1-}\right) \\
& \varepsilon\left(x, t^{n+1}\right)=\varepsilon_{1}\left(x, t^{n+1-}\right)+\varepsilon_{2}\left(x, t^{n+1-}\right) .
\end{aligned}
$$


REMARK 1. The first step is clearly a relaxation phase, as 1 t is equivalent to the solution of the following ODE problem for $t \geq t^{n}$

$$
\mid \begin{aligned}
& d_{t} \rho^{\lambda}=0 \\
& d_{t} \rho^{\lambda} u^{\lambda}=0 \\
& d_{t} E_{1}^{\lambda}=\lambda \rho^{\lambda}\left(\varepsilon_{2}^{\lambda}-\phi\left(\rho^{\lambda}, \varepsilon_{1}^{\lambda}\right)\right) \\
& d_{t} \rho^{\lambda} \varepsilon_{2}^{\lambda}=-\lambda \rho^{\lambda}\left(\varepsilon_{2}^{\lambda}-\phi\left(\rho^{\lambda}, \varepsilon_{1}^{\lambda}\right)\right)
\end{aligned}
$$

with initial data at time level $t^{n}$

$$
\rho\left(x, t^{n-}\right), u\left(x, t^{n-}\right), \varepsilon_{1}\left(x, t^{n-}\right), \varepsilon_{2}\left(x, t^{n-}\right) .
$$

and to let $\lambda \rightarrow+\infty$.

We now describe the numerical method we will use for the step of evolution in time. Although our numerical results concern both one and two dimensional problems, for simplicity of presentations we shall restrict our description to one space dimension. As we are using the finite difference version of WENO schemes in [10], extensions to two and more spatial dimensiors are simply done dimension by dimension. Essentially, the two dimensional code is the one dimensional code with an outside "do loop".

We have to solve for $t^{n} \leq t<t^{n+1}$ the following system of four equations

$$
\begin{aligned}
& \partial_{t} U+\partial_{x} F(U)=0 \\
& \quad+\text { initial conditions given by }(2.19)
\end{aligned}
$$

where

$$
\begin{aligned}
& U=\left(\rho, \rho u, E_{1}, \rho \varepsilon_{2}\right)^{T}, \\
& F(U)=\left(\rho u, \rho u^{2}+p_{1},\left(E_{1}+p_{:}\right) u, \rho u \varepsilon_{2}\right)^{T} .
\end{aligned}
$$

In order to solve the ordinary differential equation

$$
\frac{d}{d t} U=L(U)
$$

where $L(U)$ is a discretization of the spatial operator, we use a third-order TVD Runge-Kutta scheme [18].

REMARK 2. We have two possibilities for the placement of the relaxation step: each Runge-Kutta inner stage or each time step. With the first example of section 3.3, we show that the two approaches give nearly identical results in accuracy. Of course the second appr zach is less costly. We thus perform all our calculations using the second approach.

We now discretize the space into uniform intervals of size $\Delta x$ and denote $x_{j}=j \Delta x$. Various quantities at $x_{j}$ will be identified by the subscript $j$.

We use the WENO procedure described in the previous subsection to obtain the spatial operator $L_{j}(U)$ which approximates $-\partial_{x} F(U)$ at $x_{j}$. We have tested several possibilities for the definition of $L(U)$ based on WENO schemes. The first one is to use a WENO Lax- Friedrichs scheme with a full characteristic decomposition. For this purpose we need to compute a Roe ma ;rix for the system (2.24) and its eigenvalues and eigenvectors. The details of this derivation are included in Appendix A.

The other possibility is to compute the first three components of the numerical flux $\hat{F}_{j+\frac{1}{2}}^{1}, \hat{F}_{j+\frac{1}{2}}^{2}, \hat{F}_{j+\frac{1}{2}}^{3}$ by using a WENO Lax-Friedrichs scheme with a decomposition on the Euler system characteristics and to obtain the last numerical flux $\hat{F}_{j+\frac{1}{2}}^{4}$ with a scalar WENO Lax Friedrichs scheme. This is possible because the first three equations of system (2.24) are independent from the last one. 
REMARK 3. We have also tried to compute the last numerical flux by using a first order scheme specially designed in order to preserve the maximum principle for $\varepsilon_{2}[11]$. But with this approach, we lose the accuracy of the high-order WENO scheme also for the other variables.

REMARK 4. In order to make comparisons in the numerical results we have also implemented a WENO Lax-Friedrichs scheme with a full characteristic decomposition for a two molecular vibrating gas (see next section for a description of the related EOS). For this purpose we need a definition of the corresponding Roe average matrix. We give it in Appendix B. For the numerical comparisons for the other real gases we use a component-wise WENO Lax-Friedrichs scheme which requires only the computation of the sound velocity

$$
c=\sqrt{p_{, \rho}+p \frac{p_{, \varepsilon}}{\rho^{2}}}
$$

\section{Numerical results.}

3.1. Description of the different equations of states. We present here several equations of states which we will use in the computation. We find the second one in the paper of In [9], while the third one comes from Glaister [4]).

- Polytropic ideal gas. The equation of states for a polytropic ideal gas (also called perfect gas) is the following

$$
p(\rho, \varepsilon)=(\gamma-1) \rho \varepsilon
$$

Then we have

$$
p_{, \rho}=(\gamma-1) \varepsilon, \quad p_{, \varepsilon}=(\gamma-1) \rho .
$$

Air under normal conditions ( $p$ and $T$ moderate enough) can be considered as a perfect gas with $\gamma=7 / 5=1.4$ (approximately a mixture of two diatomic molecular species: $20 \%$ of $\mathrm{O}_{2}, 80 \%$ of $\mathrm{N}_{2}$ ).

- Two molecular vibrating gas. When the temperature increases the vibrational motion of oxygen and nitrogen molecules in air becomes important, and specific heats vary with temperatures. So that one must consider the following thermally perfect, calorically imperfect model for two molecular vibrating gas

$$
p(\rho, \varepsilon)=r \rho T(\varepsilon)
$$

where the temperature $\mathrm{T}$ is given by the implicit expression

$$
\rho \varepsilon=c_{v}^{t r} T+\rho \frac{\alpha \Theta_{v i b}}{\exp \left(\frac{\Theta_{v i b}}{T}\right)-1},
$$

with $r=287.086 \mathrm{~J} \cdot \mathrm{kg}^{-1} \cdot \mathrm{K}^{-1}, C_{v}^{t r}=r /\left(\gamma_{t r}-1\right), \gamma_{t r}=1.4, \Theta_{v i b}=10^{3} \mathrm{~K}, \alpha=r$. Then we have

$$
p_{, \rho}=r T(\varepsilon), \quad p_{, \varepsilon}=\frac{r \rho}{\varepsilon^{\prime}(T(\varepsilon))} \text {. }
$$

- Osborne model. R. K. Osborne from the Los Alamos Scientific Laboratory has developed a quite general equation of states in the following form [17]

$$
p(\rho, \varepsilon)=\frac{1}{E+\phi_{0}}\left(\zeta\left(a_{1}+a_{2} \zeta\right)+E\left(b_{0}+\zeta\left(b_{1}+b_{2} \zeta\right)+E\left(c_{0}+c_{1} \zeta\right)\right)\right)
$$


where $E=\rho_{0} \varepsilon$ and $\zeta=\frac{\rho}{\rho_{0}}-1$ and the constants $\rho_{0}, a_{1}, a_{2}, b_{0}, b_{1}, b_{2}, c_{0}, c_{1}, \phi_{0}$ depend on the material in question. The typical values for water are $\rho_{0}=10^{-2}, a_{1}=3.84 \times 10^{-4}, a_{2}=1.756 \times 10^{-3}, b_{0}=1.312 \times 10^{-2}$, $b_{1}=6.265 \times 10^{-2}, b_{2}=0.2133, c_{0}=0.5132, c_{1}=0.6761$ and $\phi_{l}=2 . \times 10^{-2}$. Then we have

$$
\begin{aligned}
& p_{, \rho}=\frac{1}{\rho_{0}\left(E+\phi_{0}\right)}\left(\left(a_{1}+2 a_{2} \zeta\right)+E\left(b_{1}-2 b_{2} \zeta+E c_{1}\right)\right), \\
& p_{, \varepsilon}=-\frac{\rho_{0}}{E+\phi_{0}} p+\frac{\rho_{0}}{E+\phi_{0}}\left(b_{0}+\zeta\left(b_{1}+b_{2} \zeta\right)+2 E\left(c_{0}+c_{1} \zeta\right)\right) .
\end{aligned}
$$

\subsection{One dimension cases.}

- Example 1. 1D Riemann problems with perfect gas.

We consider here two well-known problems which have the following Riemann type initial conditions:

$$
\mathbf{u}(x, 0)= \begin{cases}\mathbf{u}_{L} & \text { if } x<0 \\ \mathbf{u}_{R} & \text { if } x>0\end{cases}
$$

The first one is Sod's problem [22]. The initial data are

$$
\left(\rho_{L}, u_{L}, p_{L}\right)=(1,0,1),\left(\rho_{R}, u_{R}, p_{R}\right)=(0.125,0,0.1) .
$$

The second one is proposed by Lax [12] with

$$
\left(\rho_{L}, u_{L}, p_{L}\right)=(0.445,0.698,3.528),\left(\rho_{R}, u_{R}, p_{R}\right)=(0.5,0,0.571)
$$

Of course for this perfect gas situation there is no need to use the relaxation model in practice. The purpose of this test problem is to test the behavior of different relaxation models (different $\gamma_{1}$ 's) and different ways of treating the relaxed system (fully characteristic and partially characteristic for the first three equations only).

For this example, a uniform grid of 100 points are used and every 2 points are drawn in the figures.

We first give, in Table 3.1, a CPU time comparison among the traditional WENO characteristic scheme for the perfect gas, and the WENO scheme applied to the relaxation system, both with a fully characteristic decomposition and with a partially characteristic decomposition for the first three equations only. The calculation is done on a SUN Ultral workstation. We can see that while a fully characteristic decomposition is significantly more costly, the partially characteristic decomfosition is only slightly more costly than the WENO scheme applied to the original perfect gas Euler equations.

\begin{tabular}{cccc}
\hline Case & $\begin{array}{c}\text { WENO } \\
\text { with characteristic }\end{array}$ & $\begin{array}{c}\text { Relaxed WENO with } \\
\text { full characte-istic }\end{array}$ & $\begin{array}{c}\text { Relaxed WENO with } \\
\text { partial characteristic }\end{array}$ \\
\hline \hline Sod Shock & 2.28 & 3.49 & 2.91 \\
\hline Lax Shock & 3.32 & 4.93 & 4.08 \\
\hline
\end{tabular}

CPU time (in seconds) of different schemes for the Sod and Lax shock tube problems for a perfect gas.

In Figures 3.1 and 3.3, we present the comparison for the Jod's and Lax's shock tube problems, of the fifth order WENO schemes, applied directly to the perfect gas Juler equations using a characteristic decomposition, and applied to the relaxation model with $\gamma_{1}=3$ usinf only partial characteristic decomposition of the first 3 equations. We can see that the results are very close except for the slight over- and under-shoots in entropy for the relaxation model calculation. This indicates the feasibility of using the relaxation model. 

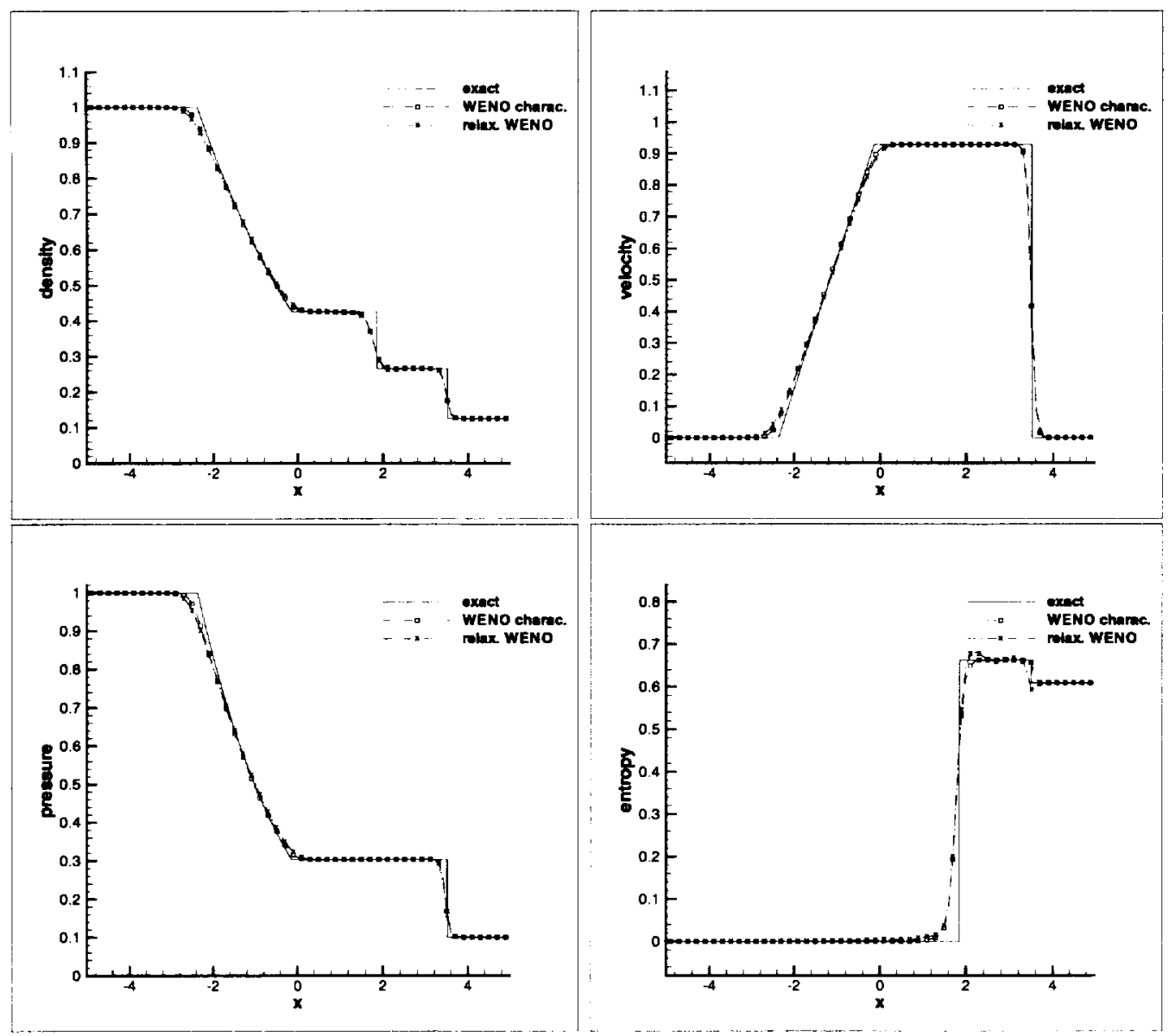

FIG. 3.1. Sod's shock tube problem with WENO-LF-5 characteristic and relaxed WENO-LF-5 partial characteristic with $\gamma_{1}=3.0$.

In Figures 3.2 and 3.4, we present the comparison for the Sod's and Lax's shock tube problems, of the fifth order WENO schemes. The top left figure compares the full characteristic decomposition for the relaxation model, with a partial characteristic decomposition for the first 3 equations only, for $\gamma_{1}=3$. We can see that the results are quite close, again indicating the feasibility of using the less costly partial characteristic decomposition for the relaxation model. The top right figure compares the effect of different $\gamma_{1}$ 's in the relaxation model. Apparently bigger $\gamma_{1}$ corresponds to larger numerical dissipation. This indicates that one should always choose the smallest possible $\gamma_{1}$ subject to stability considerations. The bottom figure compares the relaxation WENO results for $\gamma_{1}=3$ and a partial characteristic decomposition, with a component-wise WENO scheme applied directly on the original perfect gas Euler equations. Although neither uses the correct characteristic information, apparently the relaxation model results are better than the component-wise results, especially for the Lax's problem in Figure 3.4.

- Example 2. 1D Riemann problems with real gases.

In this example we compute the solutions to the Riemann shock tube problem, for the two molecular vibrating gas (3.3)-(3.5) and the Osborne model (3.6)-(3.7), with the following initial conditions in Table 3.2 .

For this example, a uniform grid of 200 points are used and every 4 points are drawn in the figures. 


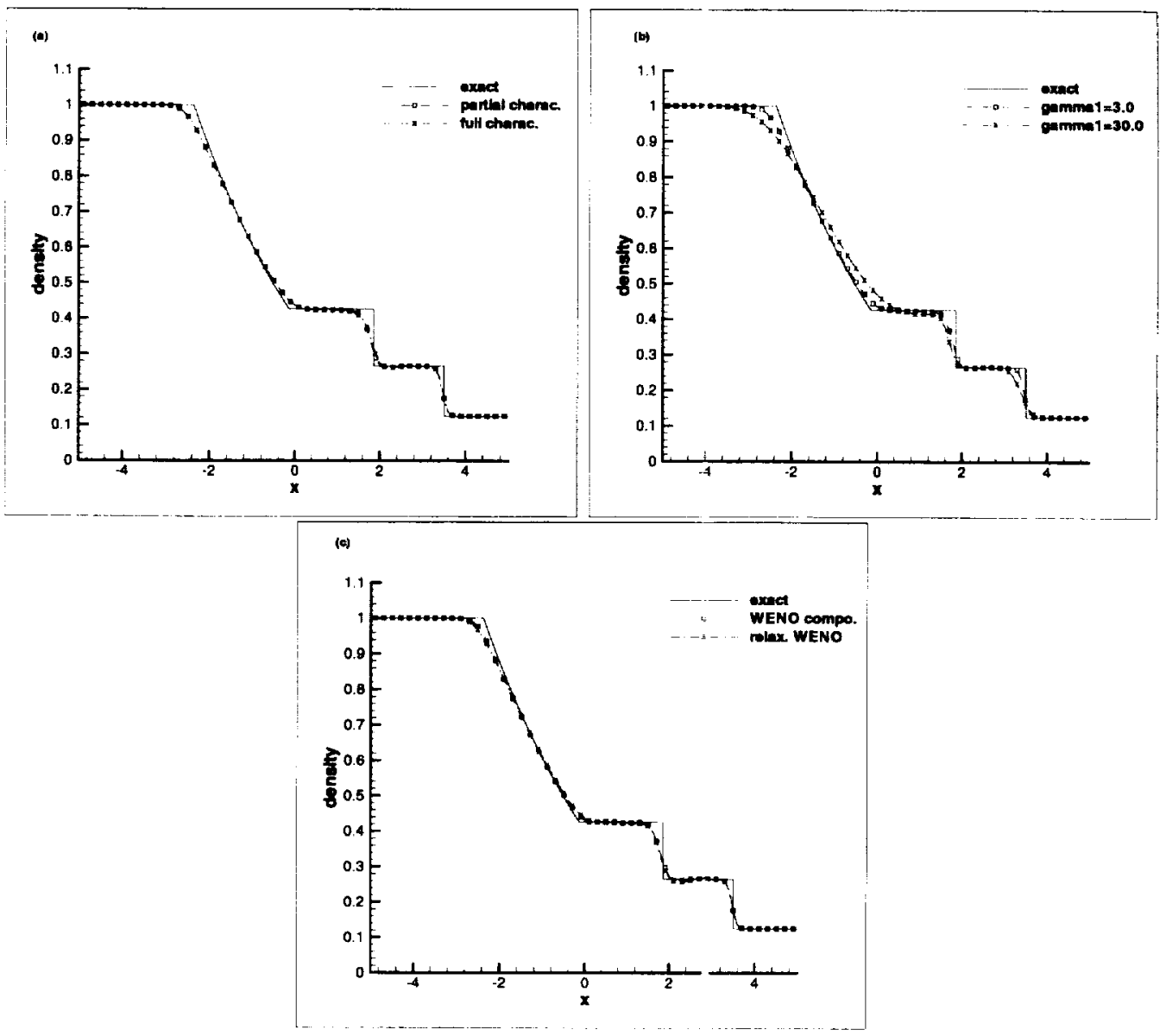

FIG. 3.2. Sod's shock tube problem with WENO-LF-5. Comparisons of partial and full characteristic decompositions for the relaxation model with $\gamma_{1}=3$ (top left); $\gamma_{1}=3$ and $\gamma_{1}=30$ for the relaxation model with partial characteristic decomposition (top right); and the relaxation model with partial characteristic decomposition with $\gamma_{1}=3$ versus the component-wise WENO applied to the original perfect gas Euler equations.

Also, the "exact solution" in the figures are obtained with the rest scheme using 2000 points.

We first give a CPU time comparison between the full chara:teristic decomposition for the original model and the partial characteristic decomposition using only the first three equations of the relaxation model, for the two molecular vibrating gas model, in Table 3.3. We can see that the partial characteristic decomposition for the relaxed model is usually more than twice less costly than the full characteristic version for the original system. Although the relaxed model has one more equation, it does not require the computation of the complicated derivatives of the EOS.

In Figure 3.5 we show the comparison of the full characteristic decomposition for the original model and the partial characteristic decomposition using only the first three equations of the relaxation model, for the two molecular vibrating gas model, with case A initial condition. The results are almost identical, indicating that the relaxation model with a partial characteristic decompe sition works well with a much reduced cost.

In Figure 3.6 we show the comparison of the component W:SNO scheme on the original system, and the partially characteristic WENO scheme on the relaxed system w:th $\gamma_{1}=2.0$, for the Osborne gas model with case A initial condition. We can see that the result of the rela ced model is much better, especially for the density. This indicates that the relaxation model is a good one for the computation of real gases. 

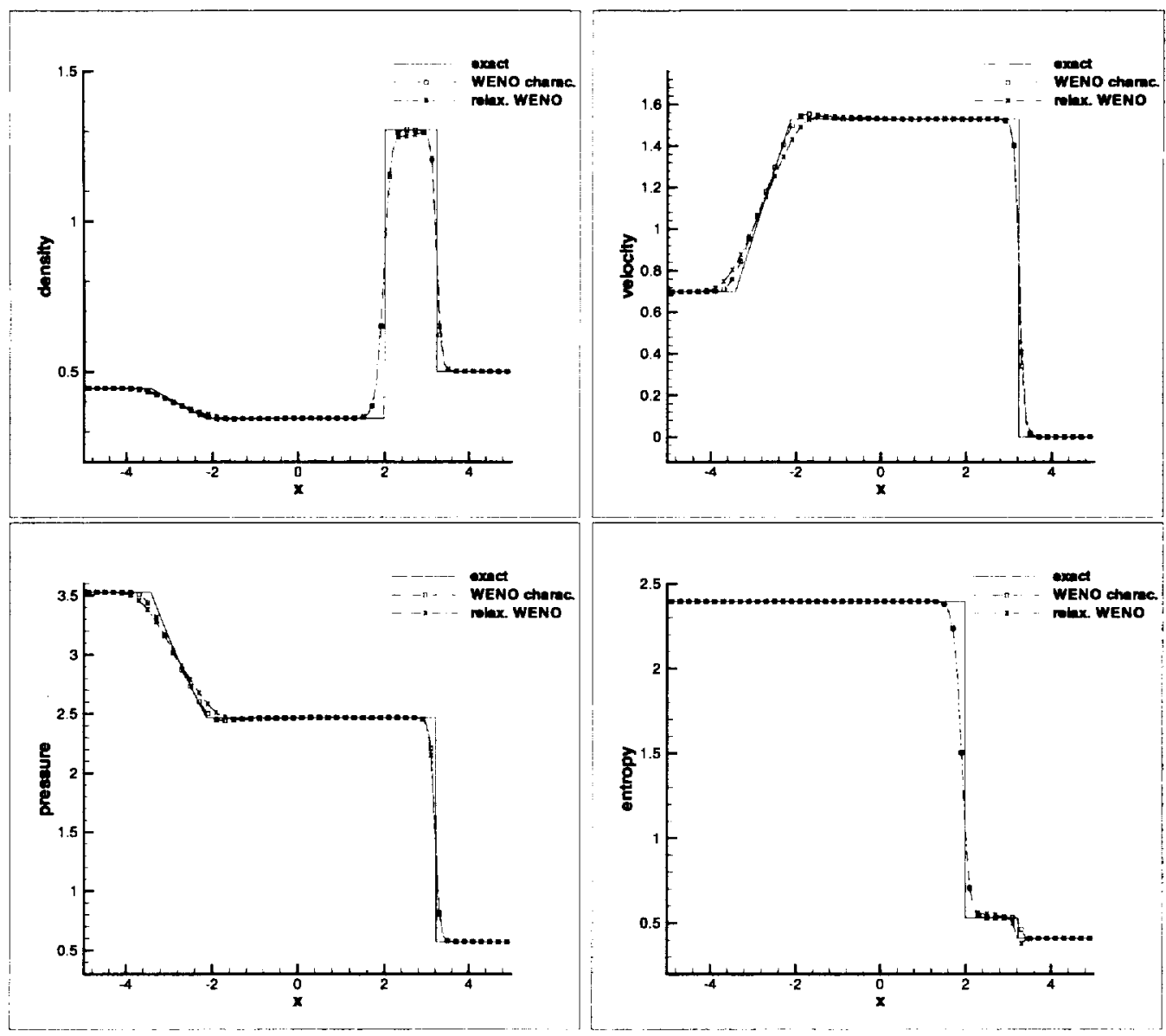

FIG. 3.3. Lax's shock tube prablem with WENO-LF-5 characteristic and relaxed WENO-LF-5 partial characteristic with $\gamma_{1}=3.0$

In Figure 3.7 we show the comparison of taking $\gamma_{1}=10$, which satisfies the stability condition (2.4), and $\gamma_{1}=2$, which satisfies only the second inequality in the stability condition (2.4), for the partial characteristic decomposition using only the first three equations of the relaxation model, and the Osborne gas model with case $\mathbf{A}$ initial condition. We can see that the $\gamma_{1}=2$ results are stable and less dissipative, indicating that in practice one does not always have to choose $\gamma_{1}$ satisfying both inequalities in condition (2.4).

We have also tested the same problems for the other initial condition cases B, C, D and E. The results are mostly similar qualitatively as in case A. To save space we will not present the results here.

\subsection{Two dimensions cases.}

- Example 3. An isentropic vortex.

This example is used to verify the accuracy of the relaxation approach, especially the placement of the relaxation steps during time stepping. The gas is ideal but we still use the relaxation model. We consider the following idealized problem for the Euler equations in 2D: the mean flow is $\rho=1, p=1$ and $(u, v)=(1,1)$ (diagonal flow). We add, to this flow, an isentropic vortex (perturbation in $(u, v)$ and the temperature $T=p / \rho$, no perturbation in the entropy $\left.S=P / \rho^{\gamma}\right)$ :

$$
(\delta u, \delta v)=\frac{\varepsilon}{2 \pi} \exp \left(\frac{1-r^{2}}{2}\right)(-\bar{y}, \bar{x}), \delta T=-\frac{(\gamma-1) \varepsilon^{2}}{8 \gamma \pi^{2}} \exp \left(1-r^{2}\right), \delta S=0
$$




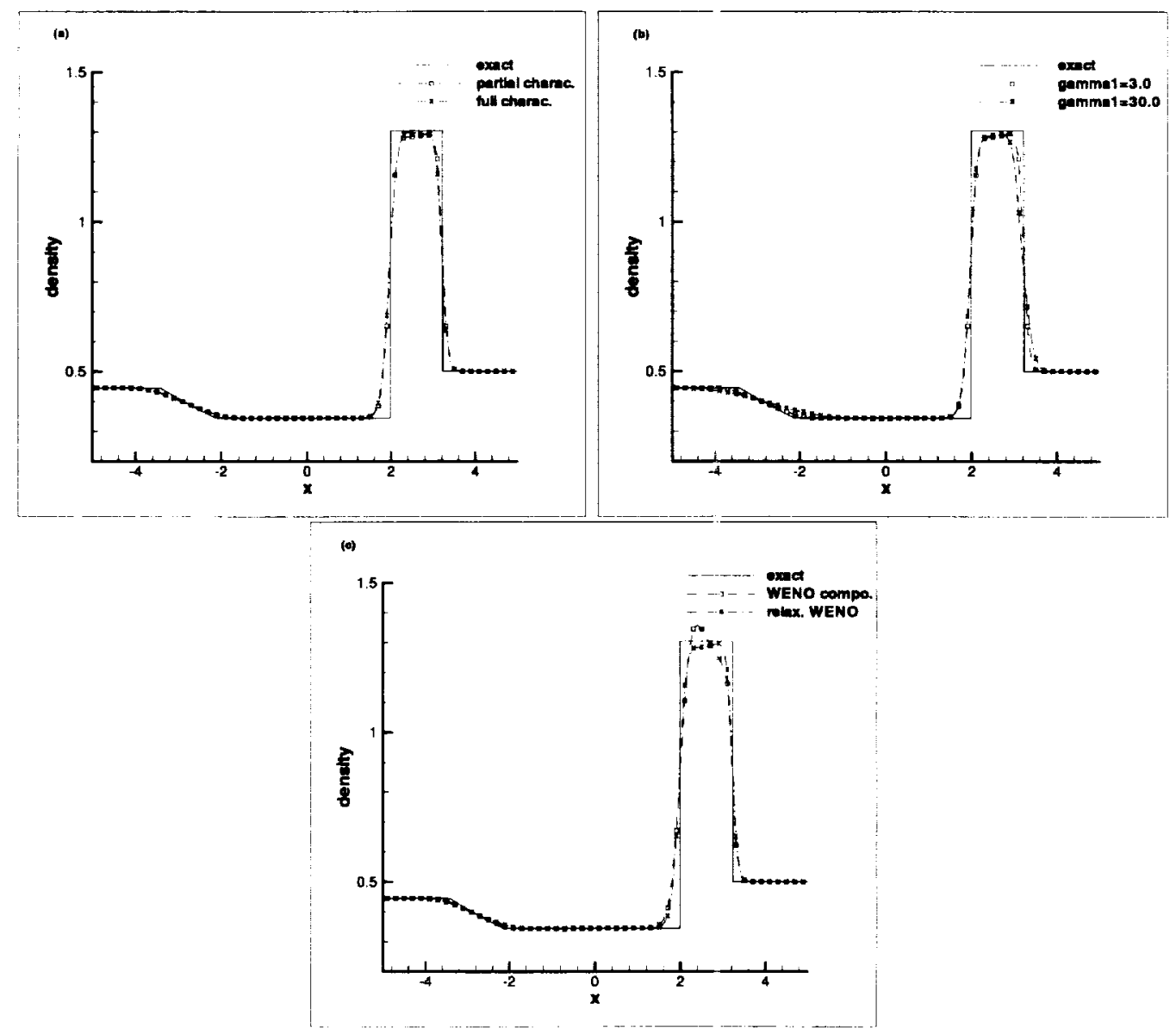

FIG. 3.4. Lax's shock tube problem with WENO-LF-5. Comparisons of partial and full characteristic decompositions for the relaxation model with $\gamma_{1}=3$ (top left); $\gamma_{1}=3$ and $\gamma_{1}=30$ for the relaxation model with partial characteristic decomposition (top right); and the relaxation model with partial characteristic decomposition urith $\gamma_{1}=3$ versus the component-wise WENO applied to the original perfect gas Euler equations.

where $(\bar{x}, \bar{y})=(x-5, y-5), r^{2}=\bar{x}^{2}+\bar{y}^{2}$, and the vortex strestgth $\varepsilon=5$. See [21].

The computational domain is taken as $[0,10] \times,[0,10]$, ex ended periodically in both directions. This allows us to perform long time simulation without having to de al with a large domain.

It is clear that the exact solution of the Euler equation with the above initial and boundary conditions is just the passive convection of the vortex with the mean velocity.

In Table 3.4 we show the accuracy result at $t=10$ (one time period). We can see that WENO for the relaxed model with $\gamma_{1}=3$ gives a somewhat larger error than $\mathrm{W}$ ENO applied directly to the original system, but the order of accuracy is correct. Moreover, to place the relazation step for each Runge-Kutta inner stage or just for each time step seems to give almost identical results. We have thus used the less costly version of putting the relaxation step for every time step in all the numerical examples in this paper.

- Example 4. Double Mach reflection.

The computational domain is chosen to be $[0,4] \times[0,1]$, altl ough only part of it $([0,3] \times[[0,1])$ is shown. The reflecting wall lies at the bottom of the computational dor sain starting from $x=1 / 6$. Initially a rightmoving Mach 10 shock is positioned at $(x, y)=(1 / 6,0)$ and nakes a $60^{\circ}$ angle with the $\mathrm{x}$-axis. For the bottom boundary, the exact post-shock condition is imposed for the part from $x=0$ to $x=1 / 6$ and a 


\begin{tabular}{llrrc}
\hline Case & State & Density & Velocity & $\begin{array}{c}\text { Specific internal } \\
\text { energy }\end{array}$ \\
\hline \hline A & Left & 0.066 & 0.0 & $7.22 \mathrm{e} 6$ \\
& Right & 0.030 & 0.0 & $1.44 \mathrm{e} 6$ \\
\hline B & Left & 1.40 & 0.0 & $2.22 \mathrm{e} 6$ \\
& Right & 0.14 & 0.0 & $2.24 \mathrm{e} 6$ \\
\hline C & Left & 1.2900 & 0.0 & $1.95 \mathrm{e} 6$ \\
& Right & 0.0129 & 0.0 & $2.75 \mathrm{e} 6$ \\
\hline D & Left & 1.00 & 0.0 & $2.00 \mathrm{e} 6$ \\
& Right & 0.01 & 0.0 & $2.50 \mathrm{e} 5$ \\
\hline E & Left & 0.01 & 2200.0 & $1.44 \mathrm{e} 5$ \\
& Right & 0.14 & 0.0 & $4.00 \mathrm{e} 5$ \\
\hline & \multicolumn{4}{c}{ TABLE 3.2 }
\end{tabular}

Initial conditions for the test cases for real gases

\begin{tabular}{ccc}
\hline Case & $\begin{array}{c}\text { WENO } \\
\text { with characteristic }\end{array}$ & $\begin{array}{c}\text { Relaxed WENO with } \\
\text { partial characteristic }\end{array}$ \\
\hline A & 12.68 & 5.21 \\
\hline B & 4.8 & 2.63 \\
\hline C & 12.53 & 4.87 \\
\hline D & 15.0 & 5.35 \\
\hline E & 15.0 & 7.84 \\
\hline
\end{tabular}

CPU time (in seconds) depending on full or partial characteristic decomposition with a two vibrating molecular gas.

reflective boundary condition is used for the rest. At the top boundary of our computational domain, the flow values are set to describe the exact motion of the Mach 10 shock. See [23] for a detailed description of this problem.

First we present the results for a perfect gas. We compare the results using WENO directly on the original system [10], and using it on the relaxed model with $\gamma_{1}=1.5$ and $\gamma_{1}=3.0$, in Figure 3.8 for a mesh of $480 \times 120$ points and Figure 3.9 for a mesh of $960 \times 240$ points. We can see that the relaxed model results are quite satisfactory, although a bigger $\gamma_{1}$ results in some small oscillations.

Next, we show the results of the same problem with the two vibrating molecular gas. The purpose here is to show that the relaxation model based algorithm does work, rather than on the details of the flow with more physical models. The results with both a $480 \times 120$ grid and a $960 \times 240$ grid are shown in Figure 3.10. Comparing with the results in [3], we can see that the main features such as the main shock being closer to the bottom boundary, and the shock below the triple point being bent, are also observed here.

4. Concluding Remarks. We have applied the fifth order WENO schemes to a relaxation model to compute the Euler equations of real gases. The algorithm does not depend on the specific form of the equation of states and does not need to compute the derivatives of the pressure law. One and two dimensional examples are shown to illustrate the accuracy and robustness of the algorithm. 

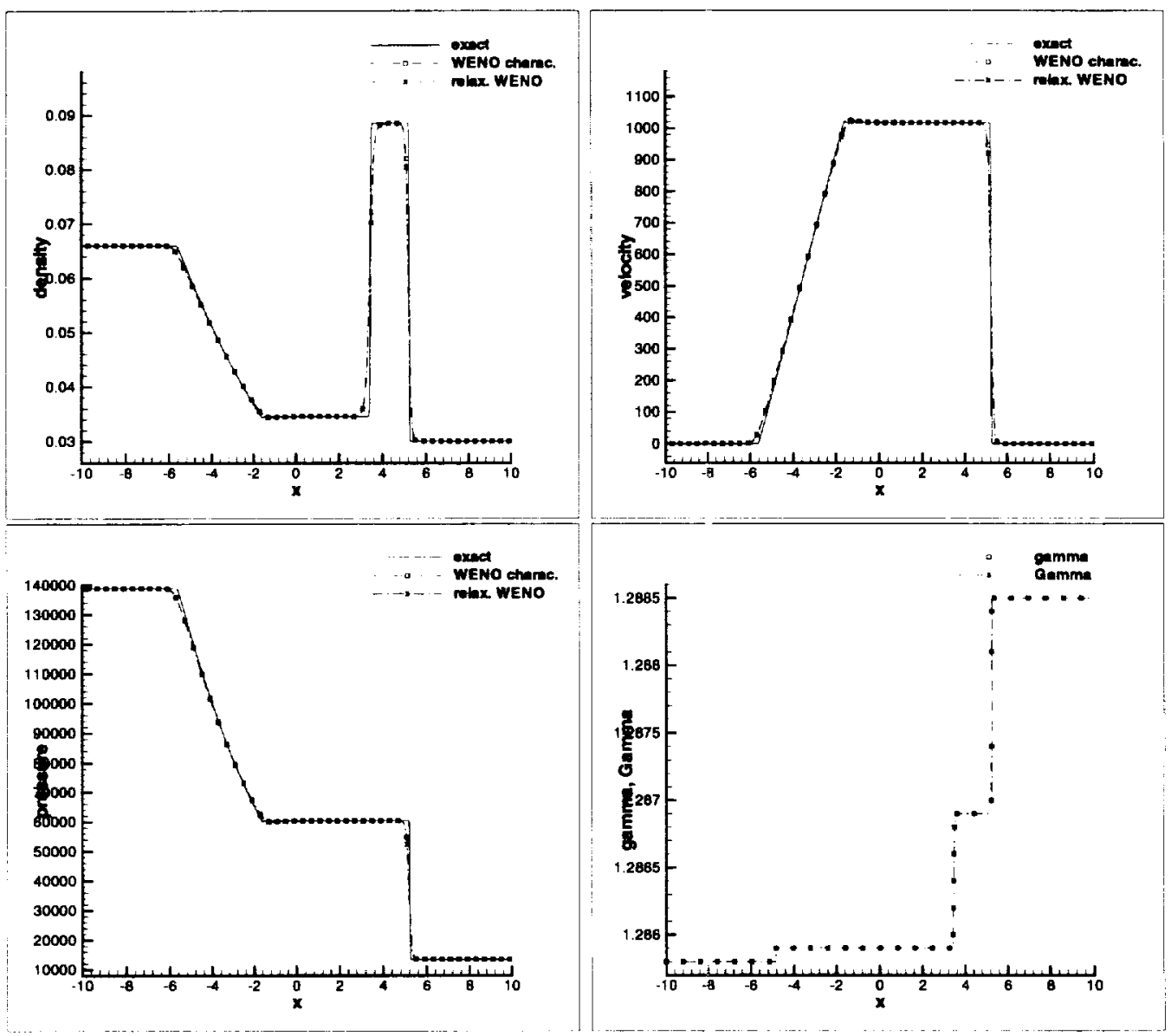

FiG. 3.5. Case A + two vibrating molecular gas model with WENO-LF-5 characteristic and relaxed WENO-LF-5 partial characteristic with $\gamma_{1}=1.5$

\begin{tabular}{|c|c|c|c|c|c|c|}
\hline \multirow[t]{2}{*}{ Nb. points } & \multicolumn{2}{|c|}{ WENO } & \multicolumn{2}{|c|}{$\begin{array}{l}\text { Relaxed WENO } \\
\text { each time step }\end{array}$} & \multicolumn{2}{|c|}{$\begin{array}{c}\text { Relaxed WENO } \\
\text { each R-K step }\end{array}$} \\
\hline & Ll error & Accuracy & L1 error & Accuracy & L1 error & Accuracy \\
\hline $20 \times 20$ & $1.07 \mathrm{e}-2$ & & $1.22 \mathrm{e}-2$ & & $1.22 \mathrm{e}-2$ & \\
\hline $40 \times 40$ & $1.06 \mathrm{e}-3$ & 3.3 & $2.16 \mathrm{c}-3$ & 2.5 & $2.17 \mathrm{e}-3$ & 2.5 \\
\hline $80 \times 80$ & $6.50 \mathrm{e}-5$ & 4.0 & $1.77 e-4$ & 3.6 & $1.78 \mathrm{e}-4$ & 3.6 \\
\hline $160 \times 160$ & $2.09 \mathrm{e}-6$ & 4.9 & $7.57 \mathrm{e}-6$ & 4.6 & $7.60 \mathrm{e}-6$ & 4.6 \\
\hline
\end{tabular}

Acknowledgment: We would like to thank Benoit Pertham ? for bringing our attention to this problem and for helpful discussions. 

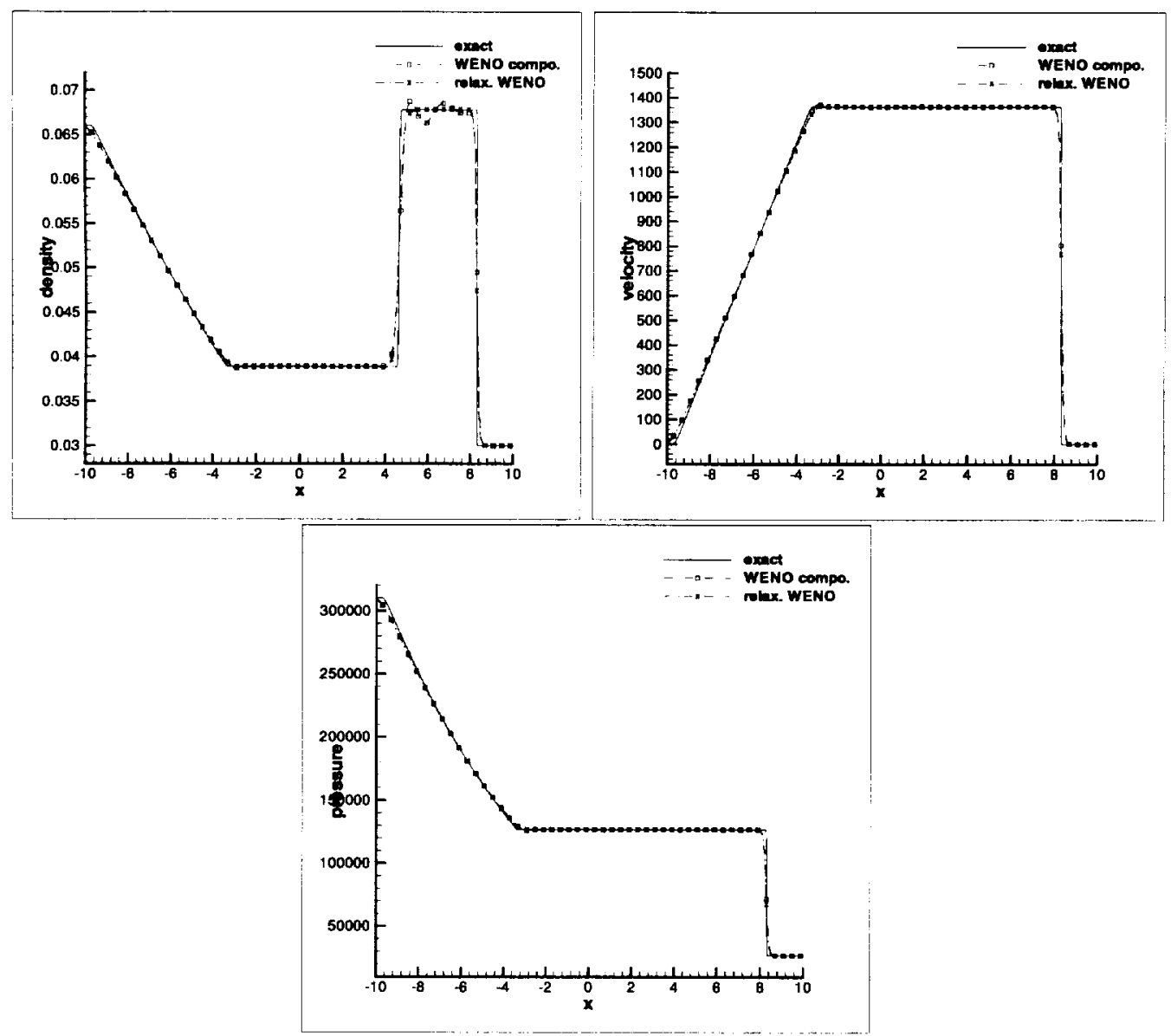

FIG. 3.6. Case $A+$ Osborne gas model with component-urse WENO-LF-5 for the original system and relaxed WENO-LF-5 partial characteristic with $\gamma_{1}=2.0$.

\section{Appendix A. Roe matrix for the relaxation system.}

Let us consider two states $U_{l}$ and $U_{r}$, then the Roe matrix for the relaxation system (2.24) is the following

$$
\bar{A}\left(U_{l}, U_{r}\right)=\left(\begin{array}{cccc}
0 & 1 & 0 & 0 \\
\left(\gamma_{1}-3\right) \frac{\bar{u}^{2}}{2} & -\left(\gamma_{1}-3\right) \bar{\rho} \bar{u} & \left(\gamma_{1}-1\right) & 0 \\
\bar{u}\left(-\bar{H}_{1}+\left(\gamma_{1}-1\right) \frac{\bar{u}^{2}}{2}\right) & \bar{H}_{1}-\left(\gamma_{1}-1\right) \bar{u}^{2} & \gamma_{1} \bar{u} & 0 \\
-\bar{\varepsilon}_{2} \bar{u} & \bar{\varepsilon}_{2} & 0 & \bar{u}
\end{array}\right),
$$

where the averaged state $\bar{\rho}, \bar{u}, \bar{H}_{1}$ are defined by

$$
\begin{aligned}
& \bar{\rho}=\sqrt{\rho_{l}} \sqrt{\rho_{r}}, \quad \bar{u}=\alpha_{l} u_{l}+\alpha_{r} u_{r}, \\
& \bar{H}_{1}=\alpha_{l} H_{1_{l}}+\alpha_{r} H_{1_{r}}, \quad \overline{\varepsilon_{2}}=\alpha_{l} \varepsilon_{1_{l}}+\alpha_{r} \varepsilon_{1_{r}},
\end{aligned}
$$

with

$$
\begin{aligned}
& H_{1}=\left(e_{1}+p_{1}\right) / \rho, \\
& \alpha_{l}=\frac{\sqrt{\rho_{l}}}{\sqrt{\rho_{l}}+\sqrt{\rho_{r}}}, \quad \alpha_{r}=1-\alpha_{l}=\frac{\sqrt{\rho_{r}}}{\sqrt{\rho_{l}}+\sqrt{\rho_{r}}} .
\end{aligned}
$$

The four eigenvalues of $\bar{A}$ are

$$
\bar{a}_{1}=\bar{u}-\bar{c}, \quad \bar{a}_{2}=\bar{u}, \quad \bar{a}_{3}=\bar{u}+\bar{c}, \quad \bar{a}_{4}=\bar{u},
$$



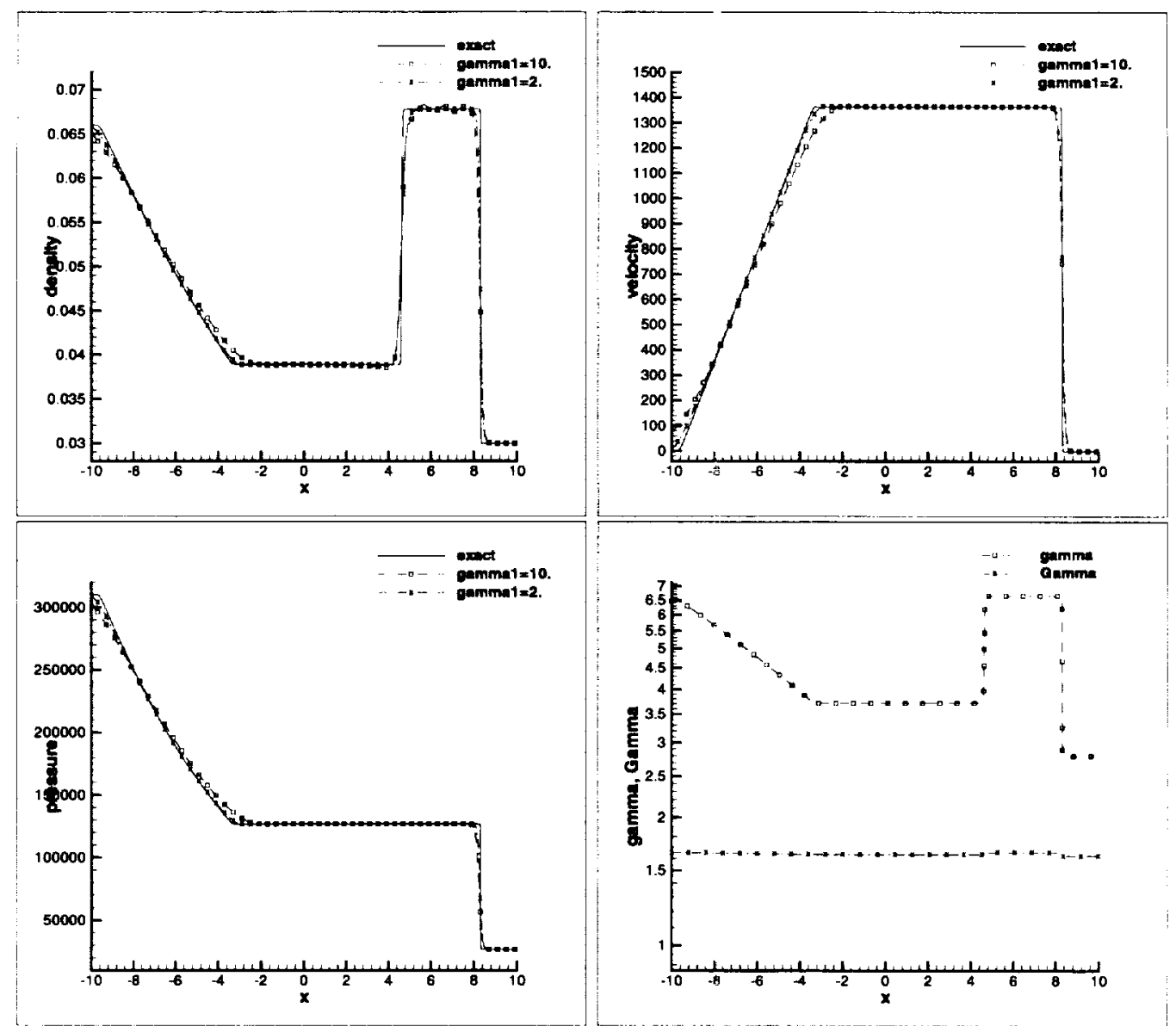

FIG. 3.7. Case A+Osborne gas model with the relaxed WENO-LF-5 fartial characteristic with $\gamma_{1}=10.0$ and $\gamma_{1}=2.0$.

where the averaged sound speed $\bar{c}$ has the usual expression

$$
\bar{c}=\sqrt{\frac{\gamma_{1} \bar{p}_{1}}{\bar{\rho}}} .
$$

A set of right eigenvectors can be

$$
\bar{r}_{1}=\left(\begin{array}{c}
1 \\
\bar{u}-\bar{c} \\
\bar{H}-\bar{u} \bar{c} \\
\bar{\varepsilon}_{2}
\end{array}\right), \bar{r}_{2}=\left(\begin{array}{c}
1 \\
\bar{u} \\
\bar{u}^{2} \\
\overline{2} \\
0
\end{array}\right), \bar{r}_{3}=\left(\begin{array}{c}
1 \\
\bar{u}+\bar{c} \\
\bar{H}+\bar{u} \bar{c} \\
\bar{\varepsilon}_{2}
\end{array}\right), \bar{r}_{4}=\left(\begin{array}{l}
0 \\
0 \\
0 \\
1
\end{array}\right) .
$$

And the corresponding orthogonal set of left eigenvectors is

$$
\bar{l}_{1}=\left(\begin{array}{c}
\frac{1}{2}\left(b_{1}+\frac{\bar{u}}{\bar{c}}\right) \\
-\frac{1}{2}\left(\bar{u} b_{2}+\frac{1}{\bar{c}}\right) \\
\frac{b_{2}}{2} \\
0
\end{array}\right), \bar{l}_{2}=\left(\begin{array}{c}
1-b_{1} \\
b_{2} \bar{u} \\
-b_{2} \\
0
\end{array}\right), \bar{l}_{3}=\left(\begin{array}{c}
\frac{1}{2}\left(b_{1}-\frac{\bar{u}}{\bar{c}}\right) \\
-\frac{1}{2}\left(\bar{u} b_{2}-\frac{1}{\bar{c}}\right) \\
\frac{b_{2}}{2} \\
0
\end{array}\right), \bar{l}_{4}=\left(\begin{array}{c}
-\bar{\varepsilon}_{2} b_{1} \\
\bar{\varepsilon}_{2} \bar{u} b_{2} \\
-\bar{\varepsilon}_{2} b_{2} \\
1
\end{array}\right)
$$



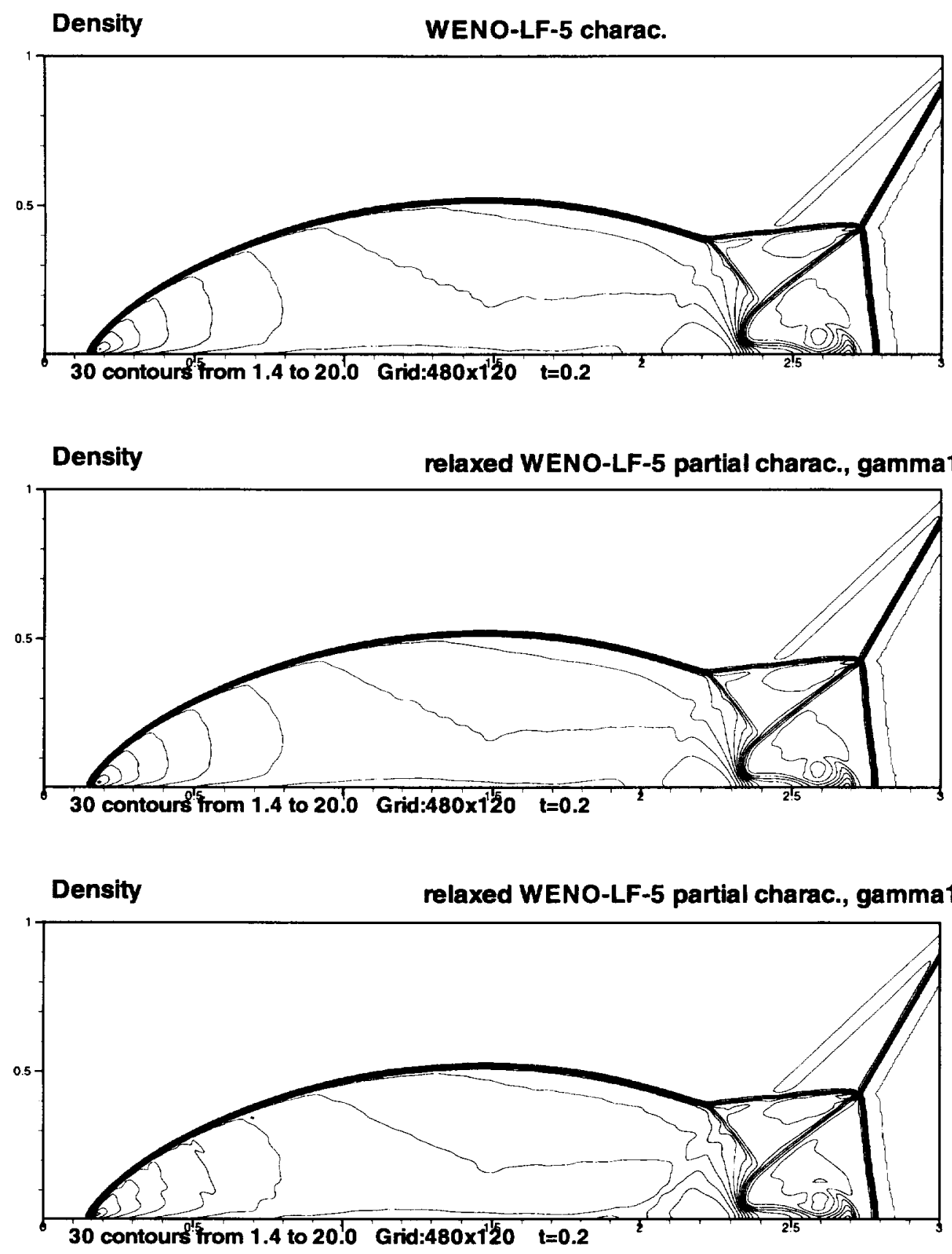

FIG. 3.8. Double-Mach reflection, perfect gas, $480 \times 120$ grid points.

where

$$
b_{1}=\frac{\left(\gamma_{1}-1\right) \bar{u}^{2}}{2 \bar{c}^{2}}, b_{2}=\frac{\left(\gamma_{1}-1\right)}{\bar{c}^{2}}
$$

Appendix B. Roe matrix for a two molecular vibrating gas.

Let us consider two states $U_{l}$ and $U_{r}$, then the Roe matrix for an Euler system of real gas is the following 

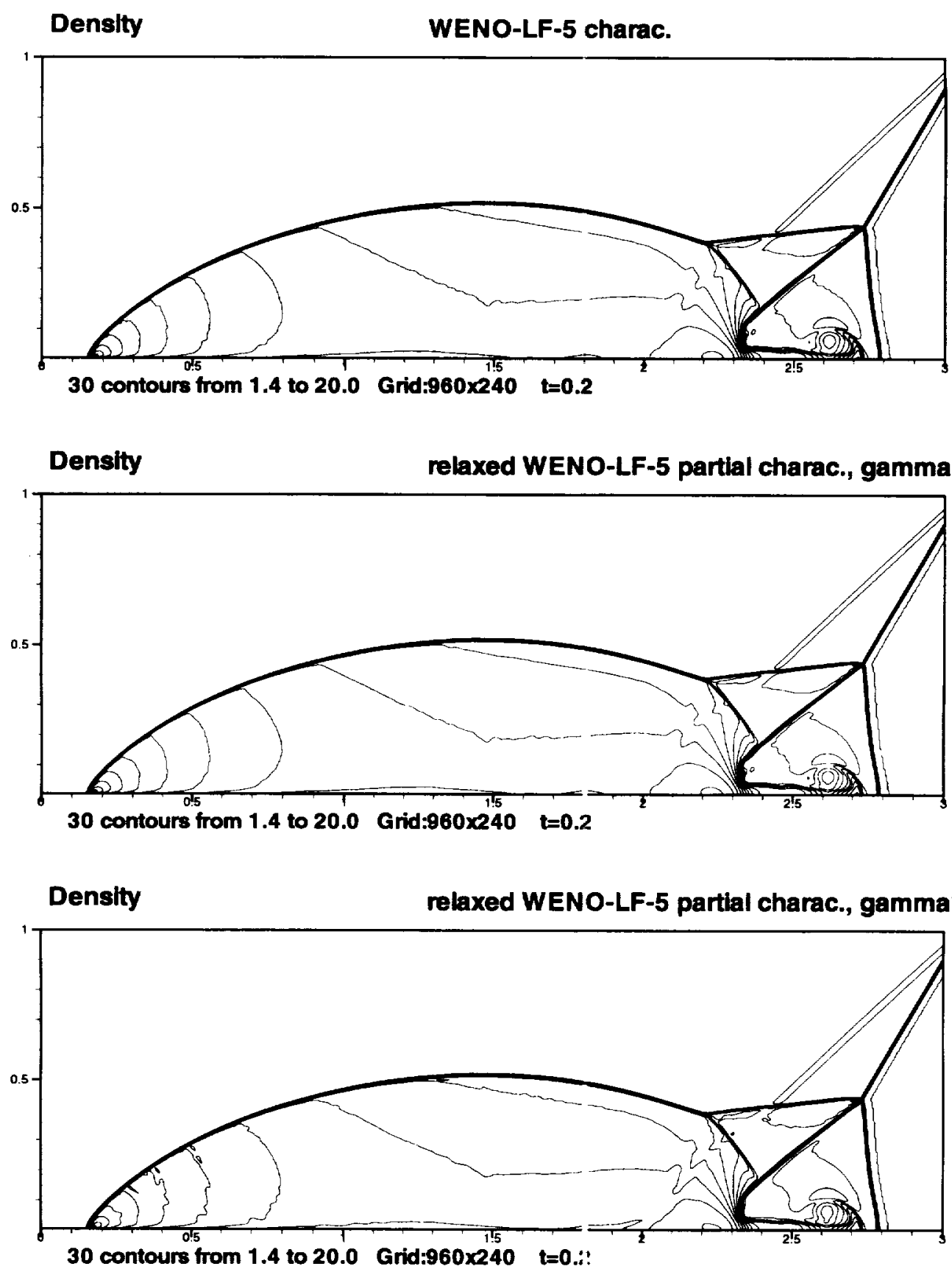

Fig. 3.9. Double-Mach reflection, perfect gas $960 \times 240$ grid points.

(see [6] for details)

$$
\bar{A}\left(U_{l}, U_{r}\right)=\left(\begin{array}{ccc}
0 & 1 & 0 \\
\bar{\chi}+\bar{\kappa} \bar{u}^{2} / 2 & (2-\bar{\kappa}) \bar{u} & \bar{\kappa} \\
\bar{u}\left(\bar{\chi}+\bar{\kappa} \bar{u}^{2} / 2-\bar{H}\right) & \bar{H}-\bar{\kappa} \bar{u}^{2} & (1+\bar{\kappa}) \bar{u}
\end{array}\right)
$$



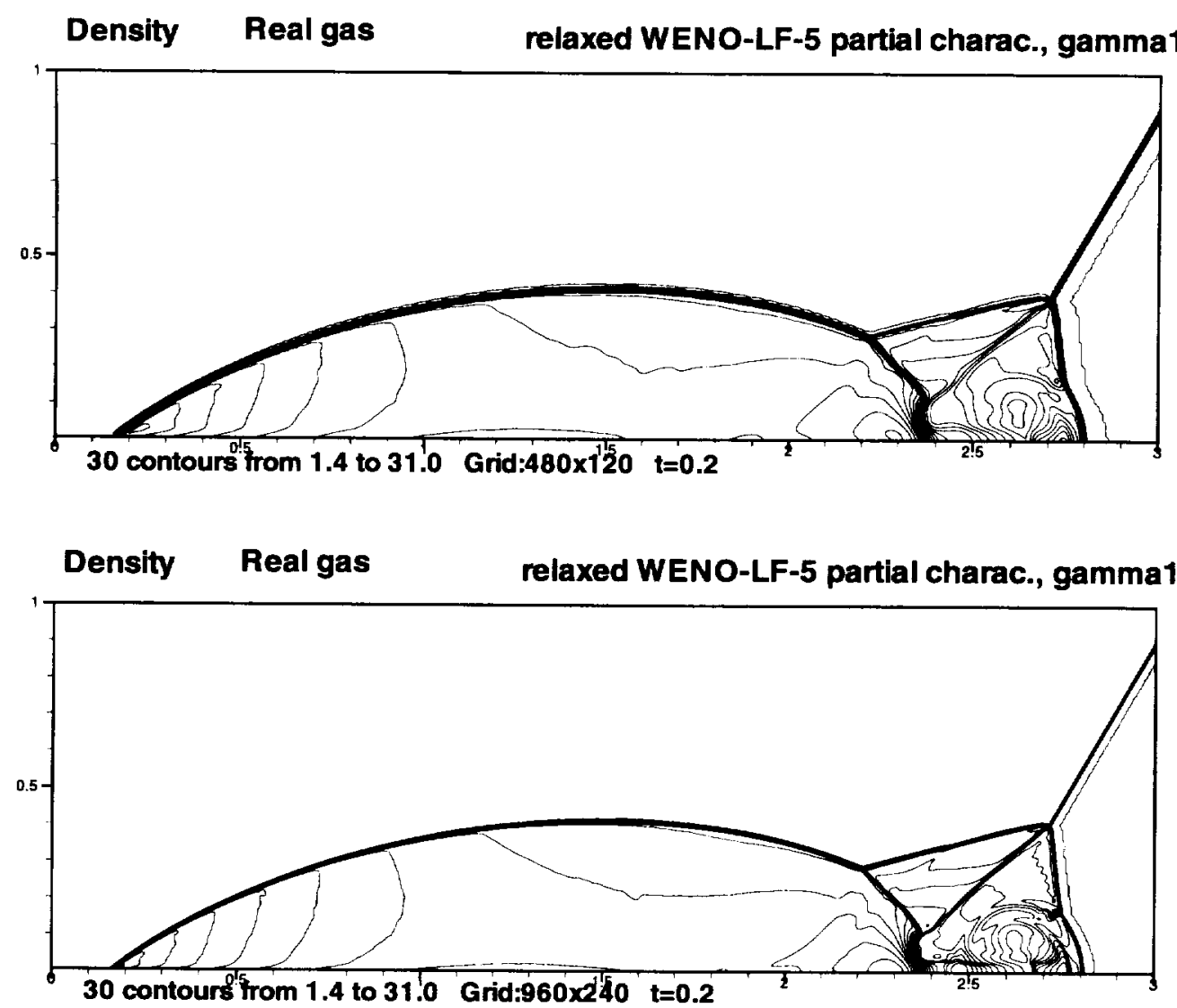

Fig. 3.10. Double-Mach reflection, two vibrating molecular gas.

where $\bar{u}, \bar{H}$ are the Roe-average values of the velocity and the total specific enthalpy $\left(H=\varepsilon+1 / 2 u^{2}+p / \rho\right)$

$$
\begin{gathered}
\bar{u}=\frac{\sqrt{\rho_{l}} u_{l}+\sqrt{\rho_{r}} u_{r}}{\sqrt{\rho_{l}}+\sqrt{\rho_{r}}}, \\
\bar{H}=\frac{\sqrt{\rho_{l}} H_{l}+\sqrt{\rho_{r}} H_{r}}{\sqrt{\rho_{l}}+\sqrt{\rho_{r}}},
\end{gathered}
$$

and $\bar{\chi}$ and $\bar{\kappa}$ are two parameters which must satisfy

$$
\Delta p=\bar{\kappa} \Delta \rho \varepsilon+\bar{\chi} \Delta \rho
$$

with $\Delta \rho=\rho_{r}-\rho_{l}, \Delta \rho \varepsilon=\rho_{r} \varepsilon_{r}-\rho_{l} \varepsilon_{l}$ and $\Delta p=p\left(\rho_{r}, \varepsilon_{r}\right)-p\left(\rho_{l}, \varepsilon_{l}\right)$.

The definitions for $\bar{\kappa}$ and $\bar{\chi}$ proposed by In [9] for a two molecular vibrating gas are

$$
\begin{aligned}
& \vec{\kappa}= \begin{cases}\frac{r\left(T\left(\varepsilon_{r}\right)-T\left(\varepsilon_{l}\right)\right)}{\varepsilon_{r}-\varepsilon_{l}} & \text { if } \varepsilon_{r} \neq \varepsilon_{l}, \\
\frac{1}{2}\left(\frac{p_{\varepsilon} \varepsilon\left(\rho_{l}, \varepsilon\right)}{\rho_{l}}+\frac{p_{\varepsilon}\left(\rho_{r}, \varepsilon\right)}{\rho_{r}}\right)=\frac{r}{\varepsilon^{\prime}(T(\varepsilon))} & \text { if } \varepsilon_{r}=\varepsilon_{l}=\varepsilon,\end{cases} \\
& \vec{\kappa}= \begin{cases}\frac{\Delta p-\bar{\kappa} \Delta \rho \varepsilon}{\Delta \rho} & \text { if } \rho_{r} \neq \rho_{l}, \\
\frac{1}{2}\left(p_{, \rho}\left(\rho, \varepsilon_{l}\right)-\frac{\varepsilon_{l}}{\rho} p_{, \varepsilon}\left(\rho, \varepsilon_{l}\right)+p_{, \rho}\left(\rho, \varepsilon_{r}\right)-\frac{\varepsilon_{r}}{\rho} p_{, \varepsilon}\left(\rho, \varepsilon_{r}\right)\right) & \\
=\frac{1}{2} r\left(T\left(\varepsilon_{l}\right)-\varepsilon_{l} T^{\prime}\left(\varepsilon_{l}\right)+T\left(\varepsilon_{r}\right)-\varepsilon_{r} T^{\prime}\left(\varepsilon_{r}\right)\right) & \text { if } \rho_{r}=\rho_{l}=\rho .\end{cases}
\end{aligned}
$$


The definitions of the eigenvalues and right and left eigenvectors are easy to obtain and are omitted here.

\section{REFERENCES}

[1] P. Colella AND H. M. GlaZ, Efficient solution algorithms for the Riemann problem for real gases, J. Comput. Phys., 59 (1985), pp. 264-289.

[2] F. Coquel AND B. Perthame, Relaxation of energy and approximate Riemann solvers for general pressure laws in fluid dynamics equations, SIAM J. Numer. Anal., to appear.

[3] R.L. Deschambault and 1.I. Glass, An update on non-stationary oblique shock-wave reflections: Actual isopycnics and numerical experiments, J. Fluid Mech., 131 (1983), pp. 27 -57.

[4] P. Glaister, An efficient numerical method for compressible flows of a real gas using arithmetic averaging, Comput. Math. Appl., 28 (1994), pp. 97-113.

[5] —_, An analysis of averaging procedures in a Riemann solver for compressible flows of a real gas, Comput. Math. Appl., 33 (1997), pp. 105-119.

[6] E. GodlewsKi AND P.-A. RaViaRT, Numerical approximation of hyperbolic systems of conservation laws, Springer, 1996.

[7] B. Grossman and R. W. Walters, Analysis of flux-split algorithms for Euler's equations with real gases, AIAA J., 27 (1989), pp. 524-531.

[8] A. Harten, B. Engquist, S. Osher and S. Chakravarthy, Uniformly high order essentially nonoscillatory schemes, III, J. Comput. Phys., 71 (1987), pp. 231-303.

[9] A. IN, Numerical evaluation of an energy relaxation method for inviscid real fluids, EDF research report HT-13/97/032/A, submitted for publication.

[10] G.-S. JIANG AND C.-W. SHU, Efficient implementation of weighed ENO schemes, J. Comput. Phys., 126 (1996), pp. 202 228.

[11] B. LARRouturou, How to preserve the mass fractions positivity when computing compressible multicomponent flows, J. Comput. Phys., 95 (1991), pp. 59-84.

[12] P. D. LAX, Weak solutions of non-linear hyperbolic equations and their numerical computations, Commmun. Pure Appl. Math., 7 (1954), pp. 159 - 193.

[13] M. S. Liou, B. van Leer, And J.-S. Shuen, Splitting of inviscid fluxes for real gases, J. Comput. Phys., 87 (1990), pp. 1-24.

[14] X.-D. Liu, S. Osher, And T. Chan, Weighed essentially non-oscillatory schemes, J. Comput. Phys., 115 (1994), pp. 200-212.

[15] C.-Y. Loh AND M. S. Liou, Lagrangian solution of supersonic real gas flows, J. Comput. Phys., 104 (1993), pp. 150-161.

[16] J.-L. MontAGnÉ, H. C. YeE, AND M. VinokUR, Comparative study of high-resolution shock-capturing schemes for a real gas, AIAA Journal, 27 (1989), pp. 1332-1346.

[17] T. D. RINEY, Numerical evaluation of hypervelocity impc ct phenomena, in High-velocity impact phenomena, R. Kinslow, ed., Academic Press, 1970, ch. V pp. 158-212.

[18] C.-W. SHU AND S. OsHER, Efficient implementation of essentially non-oscillatory shock-capturing schemes, J. Comput. Phys., 77 (1988), pp. 439-471.

[19] C.-W. ShU AND S. Osher, Efficient implementation of essentially non-oscillatory shock-capturing schemes, II, J. Comput. Phys., 83 (1989), pp. 3278. 


\begin{tabular}{|c|c|c|c|}
\hline \multicolumn{3}{|c|}{ REPORT DOCUMENTATION PAGE } & $\begin{array}{l}\text { Form Approved } \\
\text { OMB No. } 0704-0188\end{array}$ \\
\hline \multicolumn{4}{|c|}{ 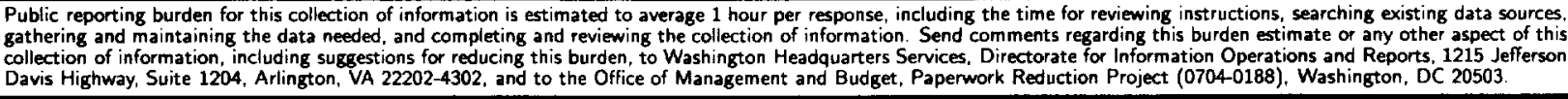 } \\
\hline 1. AGENCY USE ONLY(Leave blank) & $\begin{array}{l}\text { 2. REPORT DATE } \\
\text { September } 1998\end{array}$ & \multicolumn{2}{|c|}{$\begin{array}{l}\text { 3. REPORT TYPE AND DATES COVERED } \\
\text { Contractor Report }\end{array}$} \\
\hline \multicolumn{3}{|c|}{$\begin{array}{l}\text { 4. TITLE AND SUBTITLE } \\
\text { Real gas computation using an energy relaxation method and high- } \\
\text { order WENO schemes }\end{array}$} & \multirow[t]{2}{*}{$\begin{array}{l}\text { 5. FUNDING NUMBERS } \\
\text { C NAS1-97046 } \\
\text { WU } 505-90-52-01\end{array}$} \\
\hline \multicolumn{3}{|l|}{$\begin{array}{l}\text { 6. AUTHOR(S) } \\
\text { Philippe Montarnal } \\
\text { Chi-Wang Shu }\end{array}$} & \\
\hline \multicolumn{3}{|c|}{$\begin{array}{l}\text { 7. PERFORMING ORGANIZATION NAME(S) AND ADDRESS(ES) } \\
\text { Institute for Computer Applications in Science and Engineering } \\
\text { Mail Stop 403, NASA Langley Research Center } \\
\text { Hampton, VA } 23681-2199\end{array}$} & $\begin{array}{l}\text { 8. PERFORMING ORGANIZATION } \\
\text { REPORT NUMBER } \\
\text { ICASE Report No. } 98-42\end{array}$ \\
\hline \multicolumn{3}{|c|}{$\begin{array}{l}\text { 9. SPONSORING/MONITORING AGENCY NAME(S) AND ADDRESS(ES) } \\
\text { National Aeronautics and Space Administration } \\
\text { Langley Research Center } \\
\text { Hampton, VA } 23681-2199\end{array}$} & $\begin{array}{l}\text { 10. SPONSORING/MONITORING } \\
\text { AGENCY REPORT NUMBER } \\
\text { NASA/CR-1998-208712 } \\
\text { ICASE Report No. } 98-42\end{array}$ \\
\hline \multicolumn{4}{|c|}{$\begin{array}{l}\text { 11. SUPPLEMENTARY NOTES } \\
\text { Langley Technical Monitor: Dennis M. Bushnell } \\
\text { Final Report } \\
\text { Submitted to Journal of Computational Physics }\end{array}$} \\
\hline \multicolumn{2}{|c|}{$\begin{array}{l}\text { 12a. DISTRIBUTION/AVAILABILITY STATEMENT } \\
\text { Unclassified Unlimited } \\
\text { Subject Category } 64 \\
\text { Distribution: Nonstandard } \\
\text { Availability: NASA-CASI (301)621-0390 }\end{array}$} & & 12b. DISTRIBUTION CODE \\
\hline \multicolumn{4}{|c|}{$\begin{array}{l}\text { 13. ABSTRACT (Maximum } 200 \text { words) } \\
\text { In this paper, we use a recently developed energy relaxation theory by Coquel and Perthame and high order weighted } \\
\text { essentially non-oscillatory (WENO) schemes to simulate the Euler equations of real gas. The main idea is an energy } \\
\text { decomposition into two parts: one part is associated with a simpler pressure law and the other part (the nonlinear } \\
\text { deviation) is convected with the flow. A relaxation process is performed for each time step to ensure that the original } \\
\text { pressure law is satisfied. The necessary characteristic decomposition for the high order WENO schemes is performed } \\
\text { on the characteristic fields based on the first part. The algorithm only calls for the original pressure law once per } \\
\text { grid point per time step, without the need to compute its derivatives or any Riemann solvers. Both one and two } \\
\text { dimensional numerical examples are shown to illustrate the effectiveness of this approach. }\end{array}$} \\
\hline \multirow{2}{*}{\multicolumn{3}{|c|}{$\begin{array}{l}\text { 14. SUBJECT TERMS } \\
\text { Euler equations; real gas; weighted essentially non-oscillatory schemes }\end{array}$}} & $\begin{array}{l}\text { 15. NUMBER OF PAGES } \\
26 \\
\end{array}$ \\
\hline & & & $\begin{array}{r}\text { 16. PRICE CODE } \\
\mathrm{A} 03 \\
\end{array}$ \\
\hline $\begin{array}{l}\text { 17. SECURITY CLASSIFICATION } \\
\text { OF REPORT } \\
\text { Unclassified }\end{array}$ & $\begin{array}{l}\text { 18. SECURITY CLASSIFICATION } \\
\text { OF THIS PAGE } \\
\text { Unclassified }\end{array}$ & $\begin{array}{l}\text { 19. SECURITY CLASSIFICATION } \\
\text { OF ABSTRACT }\end{array}$ & $\begin{array}{l}\text { 20. LIMITATION } \\
\text { OF ABSTRACT }\end{array}$ \\
\hline
\end{tabular}


[20] C.-W. Shu, T.A. ZANG, G. Erlebacher, D. Whitaker, and S. Osher, High order ENO schemes applied to two- and three- dimensional compressible flow, Appl. Numer. Math., 9 (1992), pp. 45-71.

[21] C.-W. SHU, Essentially non-oscillatory and weighted essentially non-oscillatory schemes for hyperbolic conservation laws, in Advanced Numerical Approximation of Nonlinear Hyperbolic Equations, A. Quarteroni, Editor, Lecture Notes in Mathematics, CIME subseries, Springer-Verlag, to appear.

[22] G. SoD, A survey of several finite difference methods for systems of non-linear hyperbolic conservation laws, J. Comput. Phys., 27 (1978).

[23] P. WOODWARD AND P. COLELla, The numerical simulation of two-dimensional fluid flow with strong shocks, J. Comput. Phys., 54 (1984), pp. 125-160. 



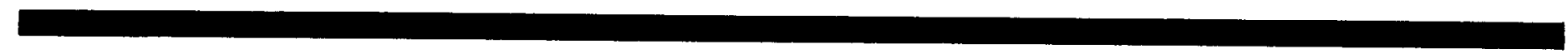

